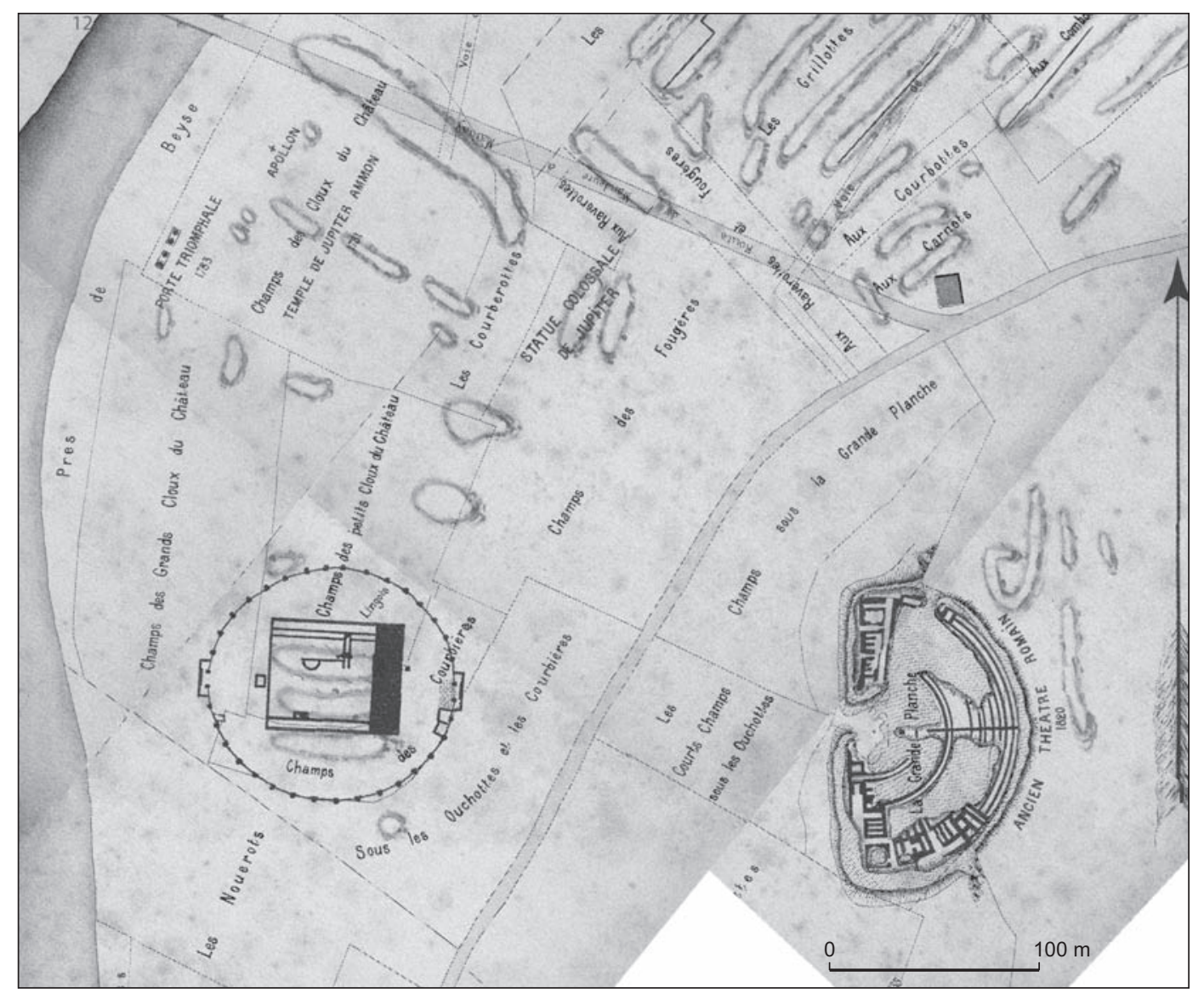

Fig. 3 - Extrait du plan de P. Barbier montrant le secteur du complexe monumental sanctuaire-théâtre de Mandeure (plan du site archéologique de Mandeure-Mathay levé en 1895 pour la Société d'émulation de Montbéliard, à 1/250, archives du musée de Montbéliard, quatre feuilles ; assemblage DAO : P. Mougin, SIVAMM).

second quartier artisanal, dit de l'Essarté, se développe à $1 \mathrm{~km}$ plus au sud, le long de la grande voie de Besançon. La zone centrale de l'agglomération, peu explorée, s'organise selon un quadrillage sensiblement orthogonal délimitant des îlots rectangulaires de tailles variables, dont l'orientation majeure (nord-ouest/sud-ouest) est imposée par la configuration du méandre. Dans la partie sud de cette zone, au lieu-dit Muraille Bourg, l'existence d'un secteur monumental, proche du complexe théâtre-sanctuaire, peut être proposée sur la base de découvertes anciennes: thermes publics et portique monumental.

L'agglomération connaît une phase majeure de développement sous les règnes de Tibère et Claude. L'activité semble florissante, de façon continue, au moins jusqu'au début du $\mathrm{III}^{\mathrm{e}} \mathrm{s}$. L'évolution de la ville entre le $\mathrm{III}^{\mathrm{e}}$ et le $\mathrm{V}^{\mathrm{e}} \mathrm{s}$. nous échappe largement. Le témoin principal des transformations majeures qui affectent le tissu et les fonctions urbaines du site au Bas-Empire consiste dans l'imposante fortification en forme de cloche édifiée à proximité du complexe sanctuaire-théâtre.

\section{ÉVOLUTION MORPHOSÉDIMENTAIRE DE LA PLAINE ALLUVIALE ET AMÉNAGEMENTS ANTHROPIQUES AUX ABORDS DU SANCTUAIRE-THÉÂTRE}

Les travaux sur la géomorphologie fluviale intégrant les apports de l'archéologie se sont multipliés depuis plusieurs années pour concerner les plaines alluviales de domaines géographiques très différents (Berger, Bravard, 2000). La reconnaissance de l'évolution morphosédimentaire de la plaine alluviale du Doubs et des traces de son occupation s'inscrit dans une thématique du programme collectif de 
recherche (PCR) consacrée à l'approche du milieu naturel et aux interactions homme/milieu. Son objectif principal est de restituer dans l'espace constitué par le territoire de l'agglomération antique d'Epomanduodurum, d'une part le contexte naturel dans lequel se situent les premières implantations humaines et, d'autre part, les modalités de transformation et d'appropriation du sol dans un espace où s'exerce une forte contrainte hydrique (Barral dir., 2001 ; Barral, 2003).

Le travail engagé porte notamment sur l'évolution du cours de la rivière qui semble s'être imposé comme un acteur essentiel de l'organisation de l'espace. La dynamique fluviale semble avoir joué, en effet, un rôle primordial dans le choix des implantions humaines, particulièrement perceptible dans la structuration de la ville gallo-romaine à l'intérieur de la boucle du Doubs (Jeannin, 1986 ; Frézouls et al., 1988 ; Mougin, 1995 ; Barral dir., 2003).

La relation homme/rivière apparaît donc au cœur de la problématique concernant l'émergence de l'agglomération d'Epomanduodurum et le développement de son territoire. Son étude a été abordée dans le cadre d'une recherche pluridisciplinaire associant plusieurs méthodes d'approche pour détecter, d'une part, les vestiges archéologiques et repérer, d'autre part, l'extension spatiale des réseaux hydrographiques anciens, comprendre leur dynamique et préciser leur durée de fonctionnement (Barral dir., 2002 et 2003). La stratégie élaborée combine les méthodes nondestructives de la prospection (carto- et photointerprétation, prospection géophysique, lever microtopographique), avec les techniques d'observation directe (forage et sondage mécaniques) et d'analyse des remplissages sédimentaires (analyses minéralogiques, paléobotaniques, datations radiométriques). Dans l'approche prospective, les méthodes géophysiques ont constitué, dès le début de ce programme, un moyen d'investigation privilégié pour la cartographie des formations géologiques superficielles et la reconnaissance des structures archéologiques enfouies.

Cet article présente les principaux résultats obtenus par les premières investigations géophysiques conduites à l'intérieur de la boucle du Doubs dans l'emprise de l'agglomération antique, aux abords du complexe monumental sanctuaire-théâtre ${ }^{3}$. Les exemples choisis illustrent la richesse des données nouvelles acquises par cette approche pluridisciplinaire dans les deux domaines qui structurent cette problématique de recherche: le fonctionnement de

3. Les auteurs de cette partie remercient L. Aubry, E. Barrès, S. Lacaze et J.-M. Valet pour leur participation active aux différentes interventions géophysiques. la plaine alluviale et la reconnaissance archéologique des traces de son occupation.

\section{CONTEXTE GÉOLOGIQUE DE LA ZONE D'ÉTUDE}

Les collines environnant la ville de Mandeure sont formées de calcaires jurassiques à structure tabulaire. Avant de déboucher dans la vallée de Mandeure, le lit du Doubs est encaissé et suit les gorges du Jura. La rivière entaille les calcaires jurassiques à partir de la ville de Mathay (pl. IV $\mathrm{n}^{\mathrm{o}} 1$, hors texte).

À Mandeure, le Doubs bute sur les calcaires plus résistants (Kimméridgien inférieur J7) de la colline du bois de Voujeaucourt et se dirige alors vers l'est pour la contourner. Il s'engage ensuite dans la large vallée d'orientation nordest/sud-ouest creusée il y a 3,4 millions d'années (Pliocène supérieur) par l'ancien fleuve alpin de l'Aar-Doubs. Cette rivière a laissé comme trace de son passage à Mandeure la formation alluviale Fx, d'origine alpine et vosgienne, composée d'éléments siliceux fixés à une altitude comprise entre $350 \mathrm{~m}$ et $400 \mathrm{~m}$ (pl. IV no 2, hors texte).

La tectonique de la plaine d'Alsace entraînant à nouveau le Rhin vers le nord, le Doubs a continué de creuser cette vallée primitive pour atteindre les marnes oxfordiennes (J4) sur lesquelles il a déposé des terrasses fluviatiles sur plusieurs niveaux. Ces terrasses sont les reliques d'anciennes surfaces de dépôt, plus ou moins retouchées par l'érosion et mises en relief par l'incision ultérieure.

La terrasse Fz est formée des alluvions fluviatiles récentes du Doubs et de ses affluents mêlant principalement des éléments calcaires et siliceux. Cette très basse terrasse domine le niveau d'étiage du Doubs et se raccorde à celui-ci par une pente douce terminée par un abrupt de $0,70 \mathrm{~m}$.

La basse terrasse Fy constitue l'élément morphologique déterminant de la plaine alluviale. Composée aussi d'alluvions calcaires, elle est épaisse de 4 à $7 \mathrm{~m}$ et culmine à une altitude moyenne de 332 à $334 \mathrm{~m}$. Dans le secteur de Pont de Roide, la fouille et l'étude du remplissage de l'abri de Rochedane (Doubs) ont permis de proposer un âge würmien final pour l'édification grossière de cette basse terrasse du Doubs (Campy, Thévenin, 1978). Postérieurement à la fin de l'Alleröd, les apports fluviatiles cessent suite à l'enfoncement du cours mineur du Doubs et à la régularisation de son débit. La morphologie de cette basse terrasse montre plusieurs ressauts qui correspondent aux entailles provoquées par des érosions mineures de l'épanchement fluviatile. La cartographie de l'extension de cette formation montre que l'agglomération antique et la 
ville actuelle de Mandeure ont été construites en grande partie sur cette basse terrasse Fy.

Un ancien méandre du Doubs, actif au Riss, d'après la notice explicative de la carte géologique sur laquelle il est représenté, venait lécher la terrasse située au nord-ouest de Mathay pour rejoindre le cours actuel au nord du pont routier de Mandeure. Il est encore marqué par une zone basse assez médiocrement drainée: le faubourg antique de Mathay est installé en travers de ce méandre (Jeannin, 1986).

\section{FONCTIONNEMENT DE LA PLAINE ALLUVIALE AUX ABORDS DU COMPLEXE SANCTUAIRE- THÉÂTRE}

\section{Problématique de RECHERCHE ET CHOIX DE LA ZONE D'ÉTUDE}

L'étude hydrographique du site réalisée par Y. Jeannin l'a conduit à formuler l'hypothèse selon laquelle le Doubs, avant de se fixer dans le méandre de son cours actuel, aurait émis vers l'est un diverticule passant au pied de la colline du théâtre avant de rejoindre plus à l'est l'église de Mandeure (Jeannin, 1986). Ce cours ancien est jalonné par une zone basse $(332 \mathrm{~m})$ partiellement inondable par grande crue. Peut-être actif aux époques protohistoriques, ce diverticule aurait été marécageux à l'époque gallo-romaine et temporairement remis en eau lors de crues importantes de la rivière, ce qui expliquerait la quasi-absence de constructions jalonnant son tracé, exception faite des rues antiques. Deux éléments semblaient appuyer cette hypothèse : d'une part l'existence d'une «digue de gros blocs» édifiée en amont, à l'embranchement de ce bras mort, pour éviter les dégâts importants occasionnés par les crues, d'autre part le changement d'orientation du parcellaire apparemment contraint par le passage de cet ancien cours du Doubs.

L'ensemble de ces informations nous a incités à cibler au sud de l'agglomération antique une première approche sur une zone englobant le complexe cultuel formé par le théâtre et le grand sanctuaire à péribole ovale. Au sud de ces deux monuments, et en aval de la digue, une vaste prairie s'étendant au lieu-dit le Champ des Ouchottes a fait notamment l'objet de l'exploration la plus poussée. Cette zone présente en effet, sur une dizaine d'hectares, des anomalies du microrelief interprétables, pour certaines, en termes de dynamique sédimentaire alluviale et, pour d'autres, en termes d'aménagements anthropiques enfouis (Barral dir., 2001 et 2002).

\section{MÉTHODES D'APPROCHE DE LA ZONE D'ÉTUDE}

La reconnaissance préliminaire de ce secteur s'est fondée principalement sur les méthodes géophysiques; ces méthodes permettent d'obtenir rapidement et de manière non destructive des informations sur la nature et la structure des matériaux du sous-sol à partir de la mesure des contrastes affectant leurs propriétés physiques (Dabas, 1998).

Une reconnaissance géophysique, associant une prospection électrique et une prospection électromagnétique, a été réalisée sur l'ensemble de la prairie. Préalablement à ces interventions, plusieurs forages mécaniques avaient été exécutés le long de transects implantés aux deux extrémités et dans la partie médiane de la prairie (Arcay et al., 2001 ; Barral dir., 2001).

\section{RECONNAISSANCE DES ÉCOULEMENTS ANCIENS}

\section{La prospection électrique}

La méthode électrique permet de caractériser les variations de la résistivité électrique apparente du sous-sol, c'est-à-dire sa capacité plus ou moins grande à conduire le courant. Une prospection électrique tractée couvrant 8 ha a été réalisée avec le dispositif multipôle ARP (Automatic Resistivity Profiling) récemment mis au point par la société Geocarta. La particularité de cet équipement est d'effectuer une mesure simultanée de la résistivité électrique du soussol à des profondeurs croissantes, correspondant respectivement à $50 \mathrm{~cm}, 1 \mathrm{~m}$ et $2 \mathrm{~m}$. La représentation des variations cartographiées selon ces trois profondeurs d'investigation permet de visualiser l'évolution des anomalies détectées avec la profondeur et d'apprécier corrélativement leur degré d'enracinement.

La planche $\mathrm{V}$ (hors texte) présente les cartes de la résistivité électrique apparente obtenues aux profondeurs de $50 \mathrm{~cm}$, $1 \mathrm{~m}$ et $2 \mathrm{~m}$. Les mesures acquises sur le terrain tous les $20 \mathrm{~cm}$ le long de profils espacés de $1 \mathrm{~m}$ ont été interpolées avec un maillage isotrope de 0,5 m par 0,5 m (Marmet et al., 2005).

Dans un contexte d'accumulation sédimentaire alluviale, l'interprétation des mesures s'appuie sur l'hypothèse, étayée par des forages mécaniques, que les matériaux sableux, limoneux et argileux recouvrent sur des épaisseurs et des dispositions variables une formation graveleuse, constituée d'éléments calcaires et siliceux. Les variations de résistivité détectées entre la surface du sol et $2 \mathrm{~m}$ de profondeur indiqueront donc principalement une variation de texture des sédiments avec des valeurs de résistivité proches de 100 ohm.m 
pour les galets et les graviers, des valeurs comprises entre $15 \mathrm{ohm} . \mathrm{m}$ et $30 \mathrm{ohm} . \mathrm{m}$ pour le sédiment limoneux et des valeurs inférieures à $10 \mathrm{ohm} . \mathrm{m}$ pour les argiles.

La représentation cartographique de ces variations permet d'individualiser tout un ensemble d'anomalies conductrices, C1, C2, C3 et C4, de résistivités inférieures à $40 \mathrm{ohm} . \mathrm{m}$, aux morphologies sinueuses, arrondies ou plus rectilignes, évocatrices de paléoformes fluviales. Ces anomalies correspondent au comblement, par des sédiments à fraction fine, limons et argile, d'anciens chenaux incisés dans la grave fluviatile. Au pied de la colline, l'anomalie C5 marque l'extension d'un recouvrement en partie colluvial correspondant à des dépôts de bas de pente, relativement fins, ayant subi un faible transport. Les anomalies R1, R2, R3 et R4, de résistivités supérieures à $50 \mathrm{ohm} . \mathrm{m}$, marquent les zones où le toit des sédiments grossiers (sables, graviers et galets) est le plus proche de la surface. L'enracinement de ces anomalies avec la profondeur est parfaitement révélé par l'évolution comparée des trois cartes de résistivité.

L'ensemble de ces observations permet de déduire un plan schématique du cours des écoulements fluviatiles anciens dans cette partie de la plaine d'inondation. D'après leur morphologie, il est possible de reconnaître les éléments d'au moins deux réseaux hydrographiques correspondant à des styles fluviaux différents :

- un premier réseau caractéristique d'un cours méandriforme de rivière, révélé devant le théâtre, par des tronçons de boucles juxtaposées, C1, C2 et vraisemblablement C3, auxquels est associé le modelé des bancs R1, R2 et R5 ;

- un second réseau à chenal unique rectiligne, C4, courant selon une direction sud-est/nord-ouest au pied du ressaut de la basse terrasse Fy.

On remarquera que l'hémicycle du théâtre, partiellement excavé dans la colline, prolonge la courbe du méandre C2, ce qui conduit à penser que le monument a vraisemblablement été implanté dans une partie du relief préalablement érodée par un cours ancien de la rivière.

\section{La prospection électromagnétique}

Une cartographie électromagnétique à large maille de la conductivité électrique apparente du sous-sol a été réalisée avec l'appareil EM-31 sur une superficie de 10 ha couvrant la totalité de la prairie. Les contrastes de conductivité que l'on mesure sont induits par des variations de lithologie, de granulométrie et de teneur en eau des sédiments (McNeill, 1992).
La planche VI n ${ }^{\circ} 1$ (hors texte) présente la cartographie de la conductivité obtenue avec des mesures acquises tous les $5 \mathrm{~m}$ le long de profils parallèles espacés de $10 \mathrm{~m}$. Dans la configuration utilisée, la profondeur d'investigation de l'appareil était proche de $6 \mathrm{~m}$. Cette cartographie électromagnétique confirme, en profondeur, l'image de la structuration du sous-sol révélée par la prospection électrique. Les anomalies de conductivité apparente, inférieures à $20 \mathrm{~ms} / \mathrm{m}$, matérialisent l'empreinte des incisions mineures dans l'épanchement fluviatile de la basse terrasse Fy et le trajet emprunté dans ce secteur par les écoulements anciens de la rivière. Au nord de la prairie, la carte précise la poursuite de l'extension du dôme de grave fluviatile R1 et du méandre C1 en face du théâtre. L'anomalie C2 montre au sud du théâtre, une conductivité très élevée, caractéristique d'une zone de très forte humidité, d'un ordre de grandeur comparable aux valeurs enregistrées au pied de la colline.

Au sud, le tracé du chenal rectiligne C4 rejoint les accumulations de limons recoupées par les carrières de graviers et à l'origine de l'alignement figurant l'ancien lit du cours d'eau repéré par Y. Jeannin.

La planche VI no 2 (hors texte) montre qu'aux contrastes dans les propriétés physiques du sol sont associés des contrastes dans le développement de la végétation induits par la teneur en humidité plus forte des sédiments fins. Les zones électriquement les plus résistantes correspondent sur le cliché de l'othophotographie IGN de 1986 à des zones claires, tandis que les zones les plus conductrices sont corrélées avec les zones les plus foncées, là où la végétation est plus dense, et nourrie grâce à un sol plus riche en argile. La planche VI $\mathrm{n}^{\circ} 3$ (hors texte) représente la profondeur estimée du toit des graviers et des galets d'après les données électriques. La modélisation géophysique surimposée à l'orthophotographie montre que dans cette partie de la terrasse alluviale, les écoulements anciens apparaissent effectivement grâce aux sédiments fins provenant des décantations consécutives aux dernières crues parvenues à leur niveau.

\section{CHRONOLOGIE ET DURÉE DE FONCTIONNEMENT DES ÉCOULEMENTS ANCIENS}

La chronologie de mise en place et la durée de fonctionnement de ces réseaux hydrographiques restaient à préciser afin d'établir des possibles relations entre des cours anciens de la rivière et des indices d'occupation repérés dans la zone d'étude.

Une analyse géomorphologique couplée à des datations au radiocarbone a donc été engagée afin de cerner les 
grandes étapes d'évolution (phases d'incisions, phases de stabilité fluviatile) dans cette partie du fond de vallée (Barral dir., 2002 et 2003).

Une tranchée de $80 \mathrm{~m}$ de long et de $2 \mathrm{~m}$ de large a été creusée à la pelle mécanique, depuis la route départementale jusqu'à la clôture du théâtre. Cette tranchée implantée perpendiculairement à l'axe de la vallée recoupait le comblement du méandre C1. Sur la coupe sud, plusieurs échantillons de sédiments ont été prélevés afin de fournir la matière sédimentaire aux datations au radiocarbone (pl. VII $\mathrm{n}^{\mathrm{o}} 1$, hors texte).

\section{Circulation des écoulements anciens}

La confrontation de la coupe de la tranchée (pl. VII $n^{\circ} 1$, hors texte) avec la cartographie de la conductivité électrique du sous-sol (pl. V et pl. VI n ${ }^{\circ} 1$, hors texte) montre qu'entre la route et le théâtre les valeurs de conductivité diminuent à l'aplomb du dôme fluviatile, là où le toit des graves de la basse terrasse (Fy) se situe le plus près de la surface $(50 \mathrm{~cm})$. Inversement, les valeurs de conductivité augmentent lorsque le toit de la grave est plus profond et recouvert par une épaisseur plus importante de limons et d'argiles.

Les hypothèses d'interprétation validées par l'ouverture de cette tranchée confortent le schéma de circulation des écoulements anciens, établi à partir des données géophysiques sur l'ensemble de la zone.

\section{Chronologie du remplissage}

Après le tamisage et le tri sous la loupe binoculaire, cinq échantillons ont été envoyés au VERA Laboratorium de Vienne (Autriche) pour datations au radiocarbone. Tous les échantillons ont été datés par AMS (Accelerator Mass Spectometry) pour compenser le déficit de matériel disponible et permettre des datations sur des échantillons de très faible volume (particules de charbons de bois comprises entre $100 \mu \mathrm{m}$ et $200 \mu \mathrm{m}$ par exemple). Ces datations radiocarbone permettent de replacer l'accumulation sédimentaire entre 9000 et $1500 \mathrm{cal}$. BP, ce qui laisse supposer un creusement du chenal principal intervenant au début de l'Holocène (Barral dir., 2002).

\section{Faciès, géométrie des dépôts et dynamique alluviale}

Les différentes étapes d'évolution pédosédimentaires de la zone d'étude sont représentées sur la planche VII $\mathrm{n}^{\text {os }} 1$ et 2 (hors texte). Chacune des étapes correspond à une nouvelle dynamique hydrologique ou un aménagement particulier du milieu par l'homme. Les datations au radiocarbone et les artefacts archéologiques retrouvés dans les différents niveaux sédimentaires permettent de discuter la chronologie de ces différentes phases d'évolution.

Le chenal du méandre C1, coulant devant le théâtre, et plusieurs couches archéologiques qui scellent son comblement ont été recoupés.

De la base au sommet du remplissage, on distingue plusieurs faciès (pl. VII $n^{\text {os }} 1$ et 2 , hors texte) :

- à l'est, le substrat calcaire du Jurassique supérieur ; à l'ouest, des blocs et galets entourés d'une matrice sableuse correspondent à l'épandage fluviatile de la basse terrasse du Doubs (Fy). Le toit de la grave se trouve sous plus de $2 \mathrm{~m}$ d'alluvions holocènes au centre de la coupe et remonte progressivement pour se retrouver seulement sous $50 \mathrm{~cm}$ de terre à l'ouest de la coupe. À l'approfondissement maximal du lit du chenal C1 correspond le contact entre le substrat calcaire et la basse terrasse Fy ;

- plusieurs niveaux argileux ou limono-argileux (16, $15,14,13)$ sont plus ou moins riches en matière organique et sont témoins d'un milieu humide de faible énergie; les périodes marquées par l'enrichissement des dépôts en matière organique (phases E-I et E-II) pourraient correspondre au développement d'une végétation de bas marais avec la présence permanente d'eau sur le site ou alors à un fort enrichissement des sédiments en résidus carbonisés. Le dépôt d'argile gris foncé (16) est daté de 7076-6828 cal. B.C., ce qui implique un creusement du chenal intervenant antérieurement à cette date. La seconde sédimentation organique, correspondant au dépôt d'argile noire à inclusions de microcharbons (13) est datée de 6643-6464 cal. B.C. Ces dates encadrent une période de relative stabilité caractérisée par un faible hydrodynamisme dans cette zone de la vallée ;

- plusieurs dépôts plus fins, limon argileux (11 et 10) et argile limoneuse (9), marquent un ralentissement des écoulements dans le chenal et le développement d'une pédogenèse qui témoigne d'un calme hydrologique relatif (phase E-III) ;

- un faciès plus sableux (8a et $8 b)$ correspond à la réactivation du chenal précédemment abandonné et partiellement comblé (phase E-IV). La dynamique hydrologique est nettement plus active et de plus grande énergie. Ce faciès est ensuite marqué par une bioturbation postdépositionnelle (nombreuses traces de racines), liée au développement ultérieur d'un sol dans les horizons supérieurs. L'échantillon, constitué de particules charbonneuses retrouvées à la base de la couche $8 \mathrm{~b}$, permet de dater post quem 
la phase E-IV, soit une reprise des flux hydriques intervenant postérieurement à 5363-5153 cal. B.C. ;

- une succession de dépôts $(7,6,5,4)$ de limon argileux et d'argile limoneuse. L'écoulement principal de la rivière est alors éloigné de la zone d'étude ; le chenal est abandonné (phase E-V). Les dépôts sont ceux d'une plaine d'inondation où alternent périodes d'inondations (apports sédimentaires plus ou moins fins) et périodes d'étiages durant lesquelles se développe une pédogenèse comme le montre l'enrichissement de la couche 4 en matière organique par rapport aux dépôts antérieurs et les traces de perturbations racinaires dans $8 \mathrm{a}$ et $8 \mathrm{~b}$. Le début de cette phase ne peut être daté plus précisément que d'avant 918-799 cal. B.C. La majorité du comblement s'effectue durant le dernier millénaire avant J.-C. ; la fin des apports est postérieure ou contemporaine de la datation 390-170 cal. B.C. ;

- un dépôt (3) définissant un mince lit de graviers, plus ou moins continu et tabulaire, semble se rattacher au niveau d'ouverture d'une fosse (F1) d'un peu plus de $1 \mathrm{~m}$ en section contenant plusieurs blocs de pierres. Il s'agit à première vue d'un niveau d'occupation. La datation 390-170 cal. B.C., effectuée au sommet du dépôt 4, et l'absence d'artefacts gallo-romains indiquent que ce niveau d'occupation est contemporain du premier âge du Fer. Il s'agirait pour le moment de la plus ancienne structure d'occupation repérée aux abords du complexe monumental théâtre/sanctuaire ;

- un ensemble formé par les niveaux $2 b, 2 c, 2 d$ et $2^{\mathrm{e}}$, dont la texture présente les caractères d'un dépôt de limon de débordement, est lié aux apports réguliers de la rivière. Cependant, les nombreux fragments de pierres calcaires observés ne peuvent être, dans ce contexte, d'origine naturelle. Il est probable que ces pierres, en position stratigraphique superficielle, correspondent à un apport anthropique postérieur au dépôt de la matrice limoneuse ;

- aux dépôts sédimentaires précédents se surimpose, d'une part, une structure excavée (fosse F2) et, d'autre part, un niveau d'accumulation sédimentaire très riche en gravillons et graviers (2a). Ces derniers dépôts remanient plusieurs fragments de tuiles gallo-romaines (phase E-VII) ;

- un horizon supérieur (1) du sol actuel correspond aux apports limoneux de débordement les plus récents et remaniés par les labours modernes.

\section{L'AMÉNAGEMENT DE LA RIVIÈRE}

Dans notre zone d'étude, différentes prospections, pédestres et subaquatiques, ont été effectuées afin d'évaluer le potentiel archéologique du lit mineur du Doubs et de reconnaître les aménagements du cours d'eau ainsi que les éventuels points de franchissement de la rivière : gués ou vestiges de ponts (Barral dir., 2003 et 2004).

La reconnaissance pédestre, en amont de l'actuel pont routier, a permis de repérer au lieu-dit la Cornaie des Isles, dans la berge de rive d'un méandre du Doubs, un alignement de très gros blocs taillés, A3 et A4, reposant sur la dalle calcaire du substrat (pl. VIII $n^{\text {os }} 1$ à 3 , hors texte). Cet alignement, localisé en amont des paléochenaux cartographiés par la géophysique, correspond au prolongement du tracé d'une digue-chemin, apparue un peu plus au sud dans la coupe d'une sablière (Jeannin, 1986). Ces blocs taillés appartiennent vraisemblablement au soubassement de cette digue édifiée pour empêcher la réactivation des chenaux lors des périodes de crue. Le creusement, plus en amont, d'un méandre au lieu-dit les Îles devant Mathay aurait pu, en rejetant le Doubs contre cette digue, saper les fondements de cet ouvrage et entraîner l'effondrement de la chaussée maçonnée dans la rivière (Jeannin, 1986).

Parallèlement à cette reconnaissance, une prospection subaquatique de la zone comprise entre cette digue de gros blocs et l'aval du pont routier actuel de Mandeure a eu lieu dans les conditions exceptionnelles de l'été 2003 (niveau de l'eau très bas et forte turbidité).

Sur toute la surface couverte par la prospection, aucun vestige archéologique n'a été trouvé dans le lit du Doubs. Le fond caillouteux est recouvert d'un dépôt calcaire blanc induré, épais de plusieurs centimètres par endroits. Les seuls objets observés sont des détritus contemporains qui, bien que déposés depuis seulement quelques décennies dans l'eau, sont déjà inclus dans l'encroûtement qui recouvre uniformément le fond de la rivière. L'examen des coupes des berges montre bien l'action érosive des crues annuelles. Ces observations laissent peu d'espoir de découvrir des vestiges archéologiques en place dans le chenal actuel.

La portion de lit mineur comprise entre le sanctuaire à péribole ovale et la fortification du Bas-Empire apparaît comme une zone de très faible hauteur d'eau, sur laquelle il pouvait être facile de traverser à gué sans aménagement de type pavage, hors période de crue bien entendu; le fond est en effet assez régulier et offre une résistance suffisante d'un bord à l'autre pour franchir la rivière (pl. IX n ${ }^{0} 1$, hors texte).

Dans cette zone, un aménagement de gros blocs taillés (A1) a été repéré dans la berge côté rive gauche, en amont du pont actuel ( $\mathrm{pl}$. IX $\mathrm{n}^{\text {os }} 2$ et 3 , hors texte). Tout comme la digue déjà observée plus en amont, aucun élément de datation ne permet d'attribuer ces probables renforcements de berge à une époque particulière. 
À une dizaine de mètres en aval de l'extrémité nord de l'alignement de blocs A1, trois bois horizontaux, manifestement travaillés, perpendiculaires à la berge côté rive gauche, ont été retrouvés ( $\mathrm{pl}$. IX n ${ }^{\circ} 4$, hors texte). Conservés dans $1 \mathrm{~m}$ d'eau, ces bois dépassaient de quelques dizaines de centimètres dans le lit du Doubs, le reste étant engagé dans la berge. Actuellement, aucun lien n'a pu être établi entre ces bois et les blocs taillés. Cependant, le lit du Doubs à cet endroit est profond de $4 \mathrm{~m}$ au maximum et à l'aplomb immédiat des blocs la tranche d'eau atteint 0,60 m à $1 \mathrm{~m}$, ce qui permettait à un bateau fluvial à fond plat de se mettre à quai à cet emplacement.

Trois prélèvements ont été effectués sur ces bois et transmis pour analyse dendrochronologique à la Société CÈDRE de Besançon (Perrault, Girardclos, 2003). Au cours de leur dégagement avant sciage, quelques tessons antiques ont été découverts dans la berge. Les trois bois, en chêne (Qercussp.), montraient pour l'échantillon 2 un aubier préservé dans son intégralité, alors que les échantillons 1 et 3 sont intégralement en bois de cœur. Le résultat de la datation, 180 apr. J.-C, validé avec un risque d'erreur quasi nul, confirme indubitablement leur rattachement à l'époque antique

S'il n'est pas possible de préciser pour l'instant à quel type de structure les trois bois découverts appartiennent (éléments d'un pont ou aménagement de quai?), on observera qu'ils se localisent au point de convergence du tracé de deux voies, repérées de part et d'autre du cours du Doubs par la prospection magnétique : l'une, en provenance du faubourg de pont selon une orientation nordouest/sud-est et l'autre, en provenance de la fortification du Bas-Empire, et orientée selon son axe médian (pl. IX n ${ }^{\circ} 1$, hors texte) (Dabas, Aubry, 1997 ; Dabas et al., 1999). En 1984, lors des fouilles de sauvetage de la tranchée du gazoduc, Y.Jeannin eut l'occasion d'observer respectivement, dans le sondage $\mathrm{Sa}$, un "pierrier antique ", interprété comme le blocage éventuel d'une pile de pont en bois et, dans le sondage $\mathrm{Sb}$, un massif maçonné de $12 \mathrm{~m}$ de long, interprété comme le tronçon d'une digue destinée à empêcher le Doubs de saper la berge de la fortification du Bas-Empire (pl. IX $\mathrm{n}^{\mathrm{o}} 1$, hors texte) (Jeannin, 1984). Ces constations incitent à placer à cet endroit un point de franchissement de la rivière (pont, bac?), sans que l'on puisse infirmer ou confirmer l'hypothèse de la présence d'une culée de pont : aucun vestige, en effet, n'est visible dans la berge en rive droite et aucun bois vertical (pieux de fondation d'une pile) n'a été à découvert. Si la structure C1, interprétée comme une «Ancienne culée d'un pont » sur le document cartographique de 1893, apparaît trop décalée par rapport à cet axe nord-ouest/sud-est, en revanche la structure C2, intitulée «Culée d'un pont» sur la carte topographique de F. Morel-Macler (1828-1847), s'aligne pratiquement sur ce tracé. Des fouilles terrestres, envisagées prochainement depuis les deux berges, ainsi que le démontage partiel de ces structures devraient permettre de lever définitivement l'indétermination concernant l'existence d'un pont à la période antique.

En aval du pont routier actuel de Mandeure, un autre alignement de blocs (A2) a été repéré sur la berge du Doubs, côté rive gauche. Il est en grande partie masqué par la végétation. Comme pour les autres alignements, aucun élément de datation n'était visible. Cet alignement (A2) correspond en fait à un renforcement de berge, déjà entrevu en 1958 sur 40 m de longueur, à la faveur de l'étiage de la rivière (Jeannin, 1986 ; Frézouls et al., 1988). Cette structure, constituée de blocs de pierres mais également de poutres, avait été repérée auparavant par Parrot au XVIII ${ }^{\mathrm{e}}$ s., qui mentionnait aussi la présence d'anneaux destinés à amarrer les bateaux (Frézouls et al., 1988).

L'identification, à hauteur de la fortification du BasEmpire, d'aménagements de berges sur les deux rives suggère que la navigabilité du Doubs à l'époque romaine commençait en amont de l'actuel pont routier de Mandeure. Notre exploration du chenal actuel, sur plus de $500 \mathrm{~m}$ en amont de ce pont, laisse penser que les aménagements portuaires de l'époque romaine, vraisemblablement rudimentaires, n'ont laissé que très peu de traces, facilement effacées par l'action érosive des crues. Dans ces conditions, la découverte des entrepôts apparaît maintenant comme le plus sûr moyen de fixer indirectement l'emplacement du port fluvial de l'agglomération antique.

\section{RECONNAISSANGE DES TRACES D'OCGUPATION AUX ABORDS DU COMPLEXE SANCTUAIRE- THÉÂTRE}

Aux abords du complexe sanctuaire-théâtre, les sources documentaires anciennes mentionnent, avec plus ou moins de précision, l'existence d'un certain nombre de vestiges (Dunod, 1709 ; Morel-Macler, 1847 ; Duvernoy, 1873 ; Jeannin, 1986). La richesse archéologique potentielle de ce secteur nous a conduits à prospecter avec les méthodes géophysiques la quasi-totalité des espaces libres de constructions, à savoir 11 ha avec la méthode magnétique et 5,6 ha avec la méthode électrique $A R P$ (voir supra, p. 362). 


\section{LA MÉTHODE MAGNÉTIQUE}

La méthode magnétique permet de mettre en évidence et de cartographier les variations du champ magnétique terrestre provoquées par des contrastes de susceptibilité magnétique propres aux matériaux constituant le sous-sol. Elle détecte des anomalies magnétiques induites par la présence de vestiges archéologiques de natures très différentes, comme les vestiges de construction, les remblais, les fossés, les zones d'activités artisanales, mais également celles provoquées par les amas de tuiles et de briques possédant une aimantation thermorémanente de forte intensité.

\section{RÉSULTATS ET INTERPRÉTATION}

\section{La zone des Ouchottes}

Les résultats de la prospection magnétique sont présentés sur la figure $4, \mathrm{n}^{\circ} 1$ et la planche $\mathrm{X}$ (hors texte) (Lacaze et al., 2002 ; Barral dir., 2004). Les variations du gradient vertical correspondent à une dynamique comprise entre $\pm 20 \mathrm{nT} / \mathrm{m}$.

Cette prospection révèle très clairement au sud du théâtre l'existence d'un ensemble totalement inédit de quatre bâtiments matérialisés par les anomalies B1, B2, B3 et B4. L'anomalie B1 dessine le plan d'une structure construite de forme rectangulaire. Ses contours intérieurs délimitent un espace de 26,50 m de large sur $31 \mathrm{~m}$ de long, au centre duquel apparaît une anomalie de forme circulaire. Les contours extérieurs dessinent de façon moins nette l'emprise de la structure $(44 \mathrm{~m} \mathrm{x} 44 \mathrm{~m})$. Les tracés internes des murs nord, sud et ouest du bâtiment montrent en leur milieu un demi-cercle, d'environ $6 \mathrm{~m}$ de diamètre, évoquant le tracé d'une abside.

Un deuxième bâtiment de forme rectangulaire, mesurant extérieurement environ $26 \mathrm{mx} 22 \mathrm{~m}$ et intérieurement $18 \mathrm{~m}$ x 13 m, est révélé par l’anomalie magnétique B2. Son allongement est orienté nord-sud, perpendiculairement à celui du bâtiment B1. L'anomalie B4, plus proche du pied du coteau, présente une forme moins aisément identifiable associant un plan rectangulaire à une structure circulaire. Son orientation, différente de celles des structures B1 et $\mathrm{B} 2$, ne permet pas d'exclure une liaison fonctionnelle avec elles.

Enfin, les vestiges d'un quatrième bâtiment de forme rectangulaire peuvent être reconnus en B3. Son orientation, comparable à celle des bâtiments B1 et B2, suggère une appartenance possible au même complexe monumental.
Plus à l'est, sur le dôme de grave fluviatile incisé par les chenaux $\mathrm{C} 1$ et $\mathrm{C} 2$, on observe un ensemble de cinq anomalies quadrangulaires (Fo), aux contours parfaitement bien définis, et de dimensions plurimétriques, correspondant sans doute à des structures excavées.

La prospection magnétique a également permis de mettre en évidence des éléments de construction insoupçonnés face au théâtre; ils apparaissent à une vingtaine de mètres environ du mur de la grande façade ouest de cet édifice, à hauteur de l'orchestra, sous la forme d'une anomalie magnétique quadrangulaire B7, de dimensions comparables (environ $8 \mathrm{~m} \times 6 \mathrm{~m}$ ) à celles de la pièce carrée située au sud du pulpitum.

Accolée à cette anomalie à fort gradient, la prospection montre une anomalie magnétique de moindre amplitude constituée de deux tronçons rectilignes clairement individualisés : un premier tronçon orienté est-ouest, dont l'une des extrémités est en contact apparent avec cette petite construction; un second tronçon orienté perpendiculairement au précédent, mais dont l'allongement n'apparaît pas parallèle à celui de la façade ouest du théâtre.

La disposition des bâtiments B1, B2 et B3 est orientée selon une direction nord-sud/est-ouest, en complète discordance avec l'orientation de la première trame viaire qui, selon un axe incliné à $45^{\circ}$ par rapport aux points cardinaux, structure l'agglomération antique (Mougin, 1995). En revanche, l'orientation est-ouest de ces bâtiments correspond à celle de l'axe monumental sur lequel s'alignent, à peu près, le grand sanctuaire à péribole ovale et le théâtre, deux éléments structurant du même grand complexe cultuel (fig. 4 et pl. X, hors texte). Les structures nouvellement découvertes sur les côtés du grand axe monumental, sans nécessairement interférer avec ces deux pôles principaux, pourraient « appartenir » à la même aire sacrée.

La forme du plan du bâtiment B1, ses dimensions et certains de ses traits architecturaux apparents, incitent à identifier cette structure à un temple et à proposer une fonction religieuse à cet édifice. La présence d'un probable conduit d'évacuation de l'eau, reconnaissable dans le tracé de l'anomalie (Co), viendrait conforter cette interprétation. Des fana sont en effet pourvus d'équipements hydrauliques, dont certains sont destinés à l'évacuation des eaux de pluies.

L'interprétation du bâtiment B2 sur la base de la forme de son plan est beaucoup moins aisée. Sa proximité par rapport au bâtiment $\mathrm{B} 1$ et son orientation comparable plaident en faveur d'un lien fonctionnel entre les deux édifices. Il pourrait s'agir dans ce cas d'un second temple 

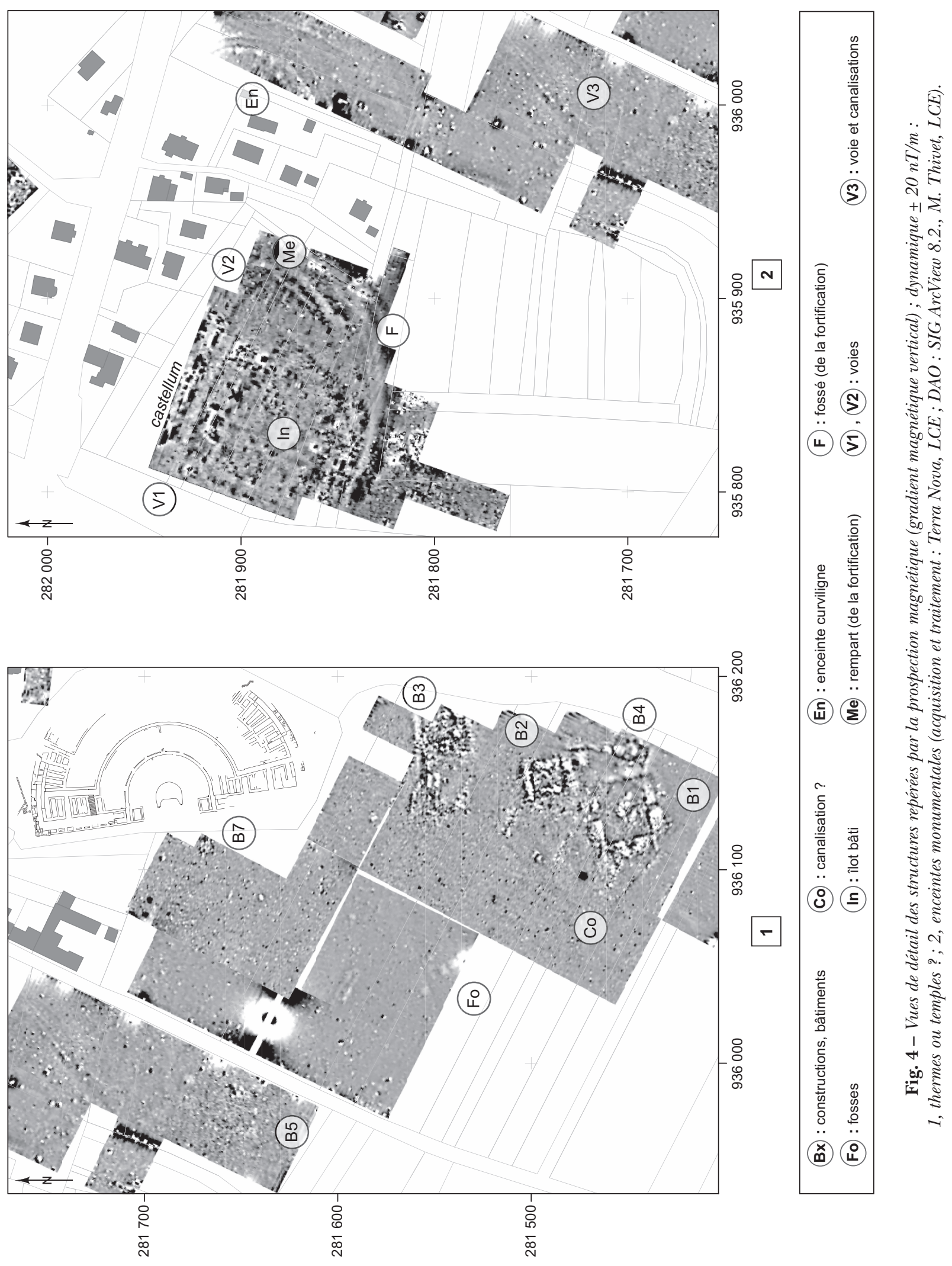
de plus petite dimension et de plan romain plus classique que le précédent, ou alors d'un simple bâtiment annexe. Dans cette hypothèse nous serions donc en présence d'un complexe cultuel de sanctuaires secondaires. La situation de Mandeure présenterait alors des analogies évidentes avec celle du site d'Avenches (Suisse), avec un espace vide de construction entre le sanctuaire et le théâtre et deux temples gallo-romains placés à l'écart de l'esplanade qui réunit les deux édifices. Une autre hypothèse, plus «en conformité " avec les sources documentaires anciennes, consisterait à voir dans ce bâtiment B1, les thermes observés au XVIII ${ }^{\mathrm{e}}$ s. par Dunod à la Maltière (Dunod, 1709) ou un monument des eaux (bassins, nymphée). Si c'était le cas, cette structure constituerait alors le troisième élément du plan architectural d'un grand complexe cultuel associant le théâtre, le grand sanctuaire à péribole ovale et un monument annexe. Les structures repérées en B3 et Co sont plus difficilement interprétables. Sans plus de certitude, elles pourraient correspondre aux vestiges dont la présence est mentionnée au sud du théâtre par différents auteurs (Morel-Macler, 1847 ; Duvernoy, 1873 ; Jeannin, 1986) et dont la fonction serait principalement en rapport avec l'alimentation (puits), le stockage (cuves) et la distribution de l'eau (aqueduc). On peut également avancer l'hypothèse d'un lien possible entre ces structures et la citerne " château d'eau », repérée plus dans la pente, sous les déblais de l'aile sud du théâtre (Jeannin, 1986).

Le bâtiment de forme rectangulaire repéré en B4 est orienté différemment des précédents. Sa localisation au pied du coteau pourrait s'expliquer par l'existence d'un aqueduc en provenance de la source de la Maltière, située plus au sud à l'extrémité de la prairie, et dont le tracé, repérable à flanc de coteau, se maintiendrait à la cote de 340 m (Jeannin, 1986).

La forme géométrique de l'anomalie B7 et sa localisation à l'arrière du mur de façade du théâtre laissent penser qu'elle pourrait correspondre à des vestiges en relation avec l'un des états de construction de cet édifice ou matérialiser des structures archéologiques encore plus anciennes.

Le plan des constructions B1, B2, B3 et B4 apparaît parfaitement lisible sur la carte de gradient magnétique vertical (fig. $4, \mathrm{n}^{\mathrm{o}} 1$ ) et beaucoup moins sur celles de la prospection électrique (pl. V, hors texte), ce qui impliquerait que le matériau de construction employé serait principalement constitué de terres cuites architecturales (briques, tuiles) possédant une aimantation thermorémanente de forte intensité. Cette hypothèse expliquerait alors le très faible contraste de résistivité électrique enregistré à l'aplomb de ces structures. Cette constatation illustre tout l'intérêt de la mise en ouvre de méthodes géophysiques mesurant des propriétés physiques différentes pour parvenir à une détection efficace des vestiges enfouis.

En revanche, la prospection électrique détecte parfaitement les anomalies de l'ensemble (Fo), sous la forme d'un alignement d'anomalies conductrices bien visibles sur le dôme de grave fluviatile R2 (pl. V et pl. X, hors texte). La nature conductrice de ces anomalies (Fo) confirme qu'il s'agit bien de structures excavées (fosses) dont l'enracinement dans le sol est attesté par la cartographie à $2 \mathrm{~m}$ de profondeur. L'interprétation de la fonction de ces fosses carrées, de grande taille (environ $4 \mathrm{~m}$ de côté), reste énigmatique. Des structures fossoyées de plus petite taille ont été également détectées sur le dôme de grave R2, à proximité de l'alignement de grandes fosses. Celui-ci se prolonge dans l'emprise du chenal pour rejoindre en contrebas du ressaut de la basse terrasse deux anomalies linéaires résistantes (murs), indexées respectivement M1 pour celle suivant la berge du chenal C2 et M2 pour celle s'alignant approximativement sur la façade du théâtre (pl. V, hors texte). L'anomalie résistante rectiligne Co, qui s'étend depuis le pied de la colline en direction du sanctuaire, correspondrait à l'élément d'un réseau hydraulique souterrain (collecteur) identique à ceux entrevus dans la coupe sud de la tranchée 2003 (pl. XI, hors texte).

$\mathrm{Au}$ nord du théâtre enfin (pl. X, hors texte), la prospection électrique $A R P$ révèle en $\mathrm{B} 6$, la poursuite des vestiges d'un habitat privé formé d'un ensemble de six à huit pièces appuyées au rocher et partiellement dégagées par J. Charrière entre 1973 à 1979 (Jeannin, 1986 ; Frézouls dir., 1988).

On remarquera qu'au sud du théâtre les vestiges de constructions se localisent principalement sur le sommet topographique du replat de la basse terrasse. Ce choix correspond sans doute à la volonté d'implanter les bâtiments hors des zones inondables en périodes de crue.

\section{La zone des Champs des Fougères}

Les résultats de la prospection magnétique sont présentés sur la figure $4, \mathrm{n}^{\text {os }} 1$ et 2 , et la planche $\mathrm{X}$ (hors texte) (Barrès et al., 2003 ; Barral dir., 2004). Les variations du gradient vertical représentées correspondent à une dynamique comprise entre $\pm 20 \mathrm{nT} / \mathrm{m}$.

L'ensemble noté (En) apparaît particulièrement remarquable. Il est organisé autour de deux anomalies semi-circulaires espacées d'environ $5 \mathrm{~m}$, dessinant un ovale ; la corde de l'arc extérieur de la structure mesure environ $70 \mathrm{~m}$. Le tracé de l'arc intérieur présente à intervalles à peu près 
réguliers de petites anomalies circulaires. Cette structure semble s'inscrire dans un espace délimité à l'est et au sud par une longue anomalie rectiligne.

L'ensemble V3 montre principalement des anomalies rectilignes et parallèles, orientées nord-est/sud-ouest aboutissant à l'angle nord-est du replat sur lequel est édifié le sanctuaire.

Plus au sud, entre le sanctuaire et le chemin, l'ensemble B5, très perturbé magnétiquement, associe plusieurs anomalies linéaires et deux anomalies ponctuelles de forme rectangulaire.

Les dimensions, la forme géométrique et les traits architecturaux de l'ensemble (En) suggèrent de l'interpréter comme les vestiges de substructions d'un édifice à plan circulaire ou ovalaire, de caractère monumental. La nature des concentrations de matériel observées en surface (nombreuses pierres taillées associées à des fragments de terres cuites architecturales) conforte cette hypothèse. Situé à une soixantaine de mètres à l'est de l'emprise de l'enceinte de la fortification du Bas-Empire, il ne semble pas que cet ensemble (En) puisse être mis en relation avec le site de la fortification (fig. 4, $\mathrm{n}^{\circ}$ 2). Sa localisation, qui coïncide avec la mention «STATUE DE JUPITER 1781 » sur un plan ancien de F. Morel-Macler (1847), correspond en réalité à une statue colossale de Mars en marbre, d'époque flavienne dont les fragments ont été trouvés dans un des murgers du lieu-dit Champs des Fougères (Pichot, 1995a et b ; voir infra, p. 394 et fig. 3). La fonction de cet édifice pourrait être en relation avec cette découverte; et les vestiges détectés par la géophysique appartenir alors à l'enceinte ovalaire d'un sanctuaire dont l'existence n'avait jamais été entrevue avant cette intervention.

La tranchée d'exploration ouverte en septembre 2003 (pl. XI, hors texte) montre que les anomalies de l'ensemble V3 (fig. 4, no 2) sont liées à la présence d'une voie enfouie à faible profondeur. Cette voie (voie 3) est composée d'une chaussée de graviers édifiée sur un radier de pierres, luimême superposé à un dallage recouvrant le conduit d'un collecteur pour l'écoulement des eaux (pl. XI n ${ }^{\text {os }} 2$ et 3, hors texte). Un regard était aménagé dans ce dallage pour permettre l'accès au conduit. En parfait état de conservation, ce collecteur, large de $30 \mathrm{~cm}$, est constitué de deux murs en pierres sèches, dont la face interne est parementée sur une hauteur de $70 \mathrm{~cm}$. Le fond du conduit est formé de pierres plates jointives. L'absence de colmatage permet de suivre visuellement son développement de part et d'autre des coupes de la tranchée. Au sud, son tracé est conservé intact jusqu'à la base de la plate-forme du sanctuaire. Au droit de la coupe sud de la tranchée, on observe le développement d'un second collecteur souterrain selon une direction nord-ouest/sud-est. Il est de même facture que le premier collecteur auquel il se raccorde. Le relevé topographique de leur orientation souterraine montre que l'orientation nord-est/sud-ouest du premier collecteur coïncide avec celles des anomalies magnétiques V3.

Les anomalies linéaires de l'ensemble B5 pourraient correspondre aux fondations d'un bâtiment arasé, comme le suggèrent la faiblesse de l'amplitude des anomalies magnétiques (fig. $4, \mathrm{n}^{\circ} 1$ ) et l'abondance des terres cuites architecturales et des fragments de pierres observés à cet endroit, à la surface du sol.

\section{La zone des Champs des Cloux du Château}

Les résultats de cette prospection réalisée sur l'emprise de la fortification du Bas-Empire sont représentés selon les variations du champ total ( $\mathrm{pl}$. X, hors texte) et selon celles du gradient magnétique vertical (fig. 4, no 2) (Dabas, Aubry, 1997 ; Mazimann dir., 1999; Mougin, 2000 ; Barral dir., 2002). Ces deux cartographies montrent que le fossé de la fortification $(\mathrm{F})$ se développe selon une courbe régulière et que l'espace intérieur est subdivisé en un secteur bâti au nordouest (In) et un secteur sans maçonneries notables au sud-est (V2). Le tracé du mur d'enceinte (Me) est repéré au sud-est de la zone prospectée sous la forme d'une anomalie curviligne épousant la courbe du fossé de la fortification (F).

Les structures à l'intérieur de la fortification s'organisent selon deux orientations distinctes correspondant à la présence de deux voies. L'une (V1) perpendiculaire au cours du Doubs et l'autre (V2) orientée selon un axe nordnord-est/sud-sud-ouest.

Un «îlot» (In) mesurant environ $60 \mathrm{~m}$ x $60 \mathrm{~m}$ s'adosse à l'emprise de la voie (V2), elle-même délimitée par des anomalies linéaires matérialisant le tracé des deux fossés qui la bordent. Les éléments du bâti (In), repérés à l'intérieur de la fortification du Bas-Empire, sont orientés perpendiculairement à cette voie, ce qui suggère la contemporanéité de ces deux structures. Les différences d'orientation de la voirie observées à l'intérieur de l'enceinte traduisent au moins deux états dans la mise en place de la trame urbaine.

\section{CONGLUSION}

L'acquisition par les méthodes de prospections géophysiques et par l'étude des remplissages sédimentaires d'une documentation aujourd'hui inédite sur les dynamiques 
hydrologiques dans la vallée du Doubs ouvre des perspectives très intéressantes pour les reconstructions paléoenvironnementales holocènes.

Ces investigations ont permis de mettre en évidence de nouvelles traces d'occupation protohistorique indiscutables dans cette partie du fond de la vallée du Doubs.

En ce qui concerne la taphonomie du site, on retiendra que les structures conservées dans les couches superficielles du lit majeur de la rivière le sont à l'état résiduel, ce qui rend a priori difficile, mais pas impossible, leur détection par les méthodes géophysiques de surface.

Dans le secteur du complexe monumental sanctuairethéâtre, survolé à maintes reprises à basse altitude, la reconnaissance géophysique a confirmé l'existence des structures archéologiques apparues fugitivement en prospection aérienne mais elle a révélé surtout d'autres structures dont l'existence était jusque-là insoupçonnée ou qui étaient matérialisées par de faibles concentrations de vestiges à la surface du sol.

Ces découvertes successives modifient radicalement la perception initiale que l'on avait de l'organisation spatiale des vestiges archéologiques dans notre zone d'étude. Elles posent la question de la compréhension de leur insertion dans la trame urbaine de l'agglomération à l'époque antique, à laquelle maintenant seule la fouille archéologique peut répondre en identifiant la nature et en précisant la fonction et la chronologie des structures détectées.

\section{LE SITE GAULOIS}

\section{INTRODUCTION}

La ville gallo-romaine a longtemps éclipsé le site gaulois, perçu essentiellement à travers des découvertes ponctuelles disparates et le lot d'objets trouvé au XIX ${ }^{\mathrm{e}}$ s. sous le grand temple romain, situé en vis-à-vis du théâtre. L'étude exhaustive de cet ensemble, qui n'avait jamais été réalisée, associée à celle d'autres vestiges plus modestes, conduit à réévaluer la nature et le statut de l'occupation gauloise. La réussite de la ville gallo-romaine s'explique certainement, au moins en partie, par la présence d'un centre économique et religieux déterminant un pôle de peuplement de première importance à la fin de l'âge du Fer. Mandeure fait en effet probablement partie, avec Verdun-sur-le-Doubs et Besançon, des trois principales agglomérations de plaine qui rythment le couloir de la moyenne vallée du Doubs, axe structurant du pays séquane (Barral, 2003).
La situation précise du site d'Epomanduodurum/Mandeure (fig. 5), un peu en retrait par rapport à l'axe est-ouest de la moyenne vallée du Doubs, peut-être considérée comme une anomalie, au regard des intérêts stratégiques romains, mais apparaît tout à fait logique dans le contexte territorial de l'âge du Fer. Le sanctuaire et l'agglomération gauloise d'Epomanduodurum se trouvent sur l'axe nord-sud de pénétration du massif du Jura par la haute vallée du Doubs, primordial pendant les âges des Métaux pour les échanges entre le plateau Suisse et le nord de l'Italie, d'une part, et le bassin rhénan, d'autre part (Millotte, Lambert, 1996 ; Barral et al., à paraître). Mandeure se situe par ailleurs en limite des territoires séquane et rauraque, position qui a sans doute une signification dans l'émergence d'un grand sanctuaire de territoire ${ }^{4}$.

Le site gaulois comprend trois composantes principales : dans la partie interne de la boucle du Doubs, et au nord-ouest, des témoins d'habitat (fig. 6, $\mathrm{n}^{\text {os }} 1-6$ ) ; au sudouest, en rive gauche, un espace funéraire (fig. $6, \mathrm{n}^{\circ} 11$ ) (Mougin et al., 1994 ; Barral, 1996) ; dans la partie sud-ouest du coude du Doubs, en rive droite, un sanctuaire, cour d'un complexe monumental au Haut-Empire (fig. 6, $\mathrm{n}^{\circ}$ 9). Une fréquentation du site dans les premiers temps du second âge du Fer est attestée par deux fibules de type Duchcov, trouvées anciennement. L'une provient du nord du site, Saint-Symphorien (fig. 6, no 1) (Tuefferd, 1878, pl. XVII), et une seconde est attribuée au sanctuaire (inédite, conservée au musée de Dijon : documentation Y. Jeannin). Une petite série d'objets du sanctuaire se rapporte au $\mathrm{III}^{\mathrm{e}} \mathrm{s}$. (La Tène C1), tandis que la phase qui est pour l'instant la mieux illustrée, dans le sanctuaire et la nécropole, correspond au $\mathrm{II}^{\mathrm{e}}$ s. (La Tène C2-D1).

P. B.

\section{L'HABITAT GAULOIS}

Les traces d'habitat sont, dans l'état actuel des connaissances, peu spectaculaires mais, réparties en différents points à l'intérieur du méandre du Doubs, elles constituent un ensemble topographiquement cohérent. Leur caractère discontinu est clairement lié aux aléas des recherches. Ces vestiges d'occupation précoce ont en effet été mis au jour à l'occasion de sondages ou de surveillances de travaux, dans des secteurs qui n'ont jamais fait l'objet de fouilles extensives. Entre 1959 et 1963, au lieu-dit Aux Arbues (fig. 6, $n^{\circ} 2$ ), sur un site de pied de coteau en bordure de la plaine

4. Sur le statut des Rauraques et leurs relations avec les Séquanes, voir Fichtl, 2000 et 2004, p. 104-107. 